\title{
Changes in anthropometric indices among Korean school students based on the 2010 and 2018 Korea School Health Examination Surveys
}

\author{
Moon Young Seo, \\ Shin-Hye Kim, \\ Mi Jung Park
}

Department of Pediatrics, Inje University Sanggye Paik Hospital, Seoul, Korea
Received: 19 May, 2020

Revised: 16 June, 2020

Accepted: 28 June, 2020

Address for correspondence:

Mi Jung Park

Department of Pediatrics, Inje University Sanggye Paik Hospital, 1342 Dongil-ro, Nowon-gu, Seoul 01757, Korea

Email: pmj@paik.ac.kr

https://orcid.org/0000-0002-7202-

$500 x$

Address for co-correspondence: Shin-Hye Kim

Department of Pediatrics, Inje University Sanggye Paik Hospital, 1342 Dongil-ro, Nowon-gu, Seoul 01757, Korea

Email:s2635@paik.ac.kr

https://orcid.org/0000-0003-0413$122 X$
Purpose: This study sought to identify changes in anthropometric values among Korean school students by comparing the most recent nationwide data with previous data.

Methods: Data from the Korea School Health Examination Survey 2018 were used. The study population consisted of 107,954 Korean school students ranging from the 1st grade in elementary school through the 3 rd year of high school. The difference in height and weight values for each sex and school grade was analyzed by comparison with the Korea School Health Examination Survey 2010.

Results: The mean heights of the 3 rd-year high school students were $173.8 \mathrm{~cm}$ and $160.9 \mathrm{~cm}$ for boys and girls, respectively. The mean height of the 3rd-year high school students showed no difference between 2010 and 2018 for either sex. However, the mean height of the age group from the 3rd grade in elementary school through the 1 st year in high school was significantly taller in 2018 compared to 2010 for both sexes. The mean weights of the 3rd-year high school students were $71.3 \mathrm{~kg}$ and $57.5 \mathrm{~kg}$ for boys and girls, respectively. The mean weight of students in 2018 was significantly higher compared to students in 2010 for most school grades. Conclusion: Although no significant change was found in near-final height between 2010 and 2018 among high school seniors, the mean height during the growth spurt was significantly higher in 2018 compared to 2010 . Weight was significantly higher in almost all school grades in 2018 compared to 2010.

Keywords: Anthropometry, Growth charts, Height, Weight, Body mass index, Students, Korea

\section{Highlights}

For Korean school students, the near-final height showed no difference between 2010 and 2018. The mean height during the growth spurt was higher in 2018 than in 2010. The mean weight was higher in almost all school grades in 2018 than in 2010.

\section{Introduction}

The linear growth status of pediatric adolescents undergoing a period of growth is the most basic and essential indicator of their overall health status. ${ }^{1)}$ Since 1967, the Korean National Growth Charts (KNGC) have been updated every 10 years based on data from the National Anthropometric Survey. ${ }^{2)}$ Due to consistent reports on the ongoing trends of early pubertal development among Korean adolescents, the 10-year interval for publishing growth charts might be relatively delayed in reflecting the current status of anthropometric indices in Korean adolescents. ${ }^{3,4)}$ For 3- to 18-year-old children and adolescents, the KNGC 2017 was obtained 
using National Anthropometric Survey data from 1997 and 2005.) Therefore, the KNGC 2017 may be limited in accurately reflecting the physical growth of the current Korean pediatric population.

In Korea, all students elementary through high school undergo a mandatory annual measurement at schools or at hospitals under the supervision of the Ministry of Education and the Korea Educational Environments Protection Agency. Additionally, the collected data are subsumed under the Korea School Health Examination Survey (KSHES). ${ }^{6}$

This study aimed to analyze the height, weight, and body mass index (BMI) of current Korean school students using the recently released KSHES 2018. We also compared the height and weight in the KSHES 2018 with measurements from the KSHES 2010 to investigate the change in body physique among Korean school students.

\section{Materials and methods}

\section{Study population}

The latest nationally representative data from the Korea School Health Examination Survey 2018 (1,023 schools including 108,016 Korean students from the 1st grade in elementary school through the 3rd year of high school; http:// www.schoolhealth.kr/) were used. ${ }^{6}$ The Ministry of Education conducts annual anthropometric measurements (height and weight) and health examinations (vision, hearing, blood pressure, urinalysis, blood analysis, chest radiography, etc.) for students in selected grades (the 1st and 4th grade in elementary school, the 1st year in middle school, and the 1st year in high school) every year to assess the health status of Korean school students following Article 7 of the School Health Act. These students visit medical institutions between April and July for health check-ups. ${ }^{6}$ ) The school staff measures the height and weight of the other students who are exempted from the health check-up every year. ${ }^{6}$ Using the National Education Information System, the results for each school are submitted to the Ministry of Education. Notably, the sample school provided 99.7\% raw data in 2018. ${ }^{6}$ The data generated through this process are government-designated statistics (approval number: 112002) based on Article 17 of the Statistics Act. ${ }^{6}$

This study used the publicly available data from the KSHES 2018. Of the entire study population, students whose weights were above the range created by the mean $+5 \times$ standard deviation by sex and height $(n=62)$ were excluded. The final study population comprised 107,954 Korean students from the 1st grade in elementary school through the 3rd year of high school. The KSHES 2010 result report (747 schools including 188,352 Korean students from the 1 st grade in elementary school through the 3rd year of high school; http:// www.schoolhealth.kr/) was used to compare against the 2018 students' physiques.

\section{Anthropometric measurements}

Height $(\mathrm{cm})$ was measured from the soles of the feet, after removing the shoes and placing the heels together, to the top of the student's head, with the back, hip, and heels firmly positioned against the vertical backboard of the stadiometer. In the upright position, arms were placed on the sides of the body, and eyes and ears remained in a horizontal position. Weight $(\mathrm{kg})$ was measured while wearing light clothes without shoes on a digital scale with an accuracy of $0.1 \mathrm{~kg}$. ${ }^{6}$ The BMI was calculated by dividing the weight $(\mathrm{kg})$ by the square of the height $(\mathrm{m})$.

\section{Statistical analysis}

IBM SPSS Statistics ver. 21.0 (IBM Co., Armonk, NY, USA) was used for performing statistical analyses. The sample data in the dataset were weighted for all statistical analyses. The values of the variables are presented as mean \pm standard error or numbers (weighted percentages). The complex samples crosstabs procedures were used for analyzing categorical variables, and the complex samples descriptive procedures were used for analyzing continuous variables. To analyze the mean values of height, weight, and BMI, the complex samples general linear models were used. The frequencies procedure was used to obtain summary measures for the selected percentile values of height, weight, and BMI, according to sex and school grade.

Table 1. General characteristics of the study subjects $(n=107,954)$

\begin{tabular}{|c|c|c|}
\hline Characteristic & MeantSE & No. $\left(\%{ }^{*}\right)$ \\
\hline Age (yr) & $11.9 \pm 0.00$ & \\
\hline \multicolumn{3}{|c|}{ School grade (yr) } \\
\hline \multicolumn{3}{|c|}{ Elementary school } \\
\hline Grade 1 & $6.4 \pm 0.01$ & $6,437(8.2)$ \\
\hline Grade 2 & $7.3 \pm 0.01$ & $6,537(8.1)$ \\
\hline Grade 3 & $8.4 \pm 0.01$ & $6,532(7.7)$ \\
\hline Grade 4 & $9.4 \pm 0.01$ & $6,556(8.1)$ \\
\hline Grade 5 & $10.3 \pm 0.01$ & $6,564(8.5)$ \\
\hline Grade 6 & $11.4 \pm 0.01$ & $6,465(7.9)$ \\
\hline \multicolumn{3}{|c|}{ Middle school } \\
\hline Grade 1 & $12.4 \pm 0.01$ & $9,823(7.5)$ \\
\hline Grade 2 & $13.4 \pm 0.01$ & $10,002(8.1)$ \\
\hline Grade 3 & $14.4 \pm 0.01$ & $10,325(8.4)$ \\
\hline \multicolumn{3}{|l|}{ High school } \\
\hline Grade 1 & $15.4 \pm 0.01$ & $12,027(8.2)$ \\
\hline Grade 2 & $16.3 \pm 0.01$ & $12,944(9.1)$ \\
\hline Grade 3 & $17.3 \pm 0.01$ & $13,742(10.2)$ \\
\hline \multicolumn{3}{|l|}{ Sex } \\
\hline Boys & - & $55,039(51.8)$ \\
\hline Girls & - & $52,915(48.2)$ \\
\hline \multicolumn{3}{|c|}{ Residential area } \\
\hline Urban & - & $83,680(83.6)$ \\
\hline Suburban & - & $8,431(5.9)$ \\
\hline Rural & - & $15,843(10.5)$ \\
\hline
\end{tabular}

"Weighted percentages after accounting for the sampling design. 
An independent samples $t$-test was used to compare the mean height and weight between participants in the KSHES 2010 and 2018. A $P$-value of $<0.05$ was considered statistically significant.

\section{Results}

\section{General characteristics}

Table 1 presents the weighted distribution of the participants' general characteristics. Participants included 107,954 Korean students from the 1st grade in elementary school through the 3 rd year in high school. The mean age of the overall study population was $11.9 \pm 0.00$ years, and boys accounted for $51.8 \%$ of the study population. The regional distributions of urban (major cities), suburban (small and medium-sized cities), and rural (small towns) areas were $83.6 \%, 5.9 \%$, and $10.5 \%$, respectively.

\section{Percentile values of height according to sex and school grade}

Table 2 presents the mean and percentile values of height according to sex and school grade. The 50th percentile values of the height of 6th-grade elementary school boys and girls were $151.9 \mathrm{~cm}$ and $152.4 \mathrm{~cm}$, respectively. The 50th percentile values of the height of 3rd-year high school boys and girls were 173.7 $\mathrm{cm}$ and $160.8 \mathrm{~cm}$, respectively. The $3 \mathrm{rd}$ percentile values of height, which are associated with short stature designation, were $162.9 \mathrm{~cm}$ and $151.0 \mathrm{~cm}$ for 3rd-year high school boys and girls, respectively. ${ }^{5}$ The steepest change in the median height was observed for boys between the 6th grade in elementary school

Table 2. Mean and percentile values of height by sex and school grade in Korean school students

\begin{tabular}{|c|c|c|c|c|c|c|c|c|c|c|c|c|c|c|c|c|}
\hline \multirow{2}{*}{ School grade } & \multirow{2}{*}{ No. } & \multirow{2}{*}{ Mean \pm SE } & \multirow{2}{*}{ Difference $^{*}$} & \multicolumn{13}{|c|}{ Percentile values of height (cm) } \\
\hline & & & & $1 \mathrm{st}$ & $3 r d$ & 5 th & 10th & 15th & 25th & 50 th & 75 th & 85th & 90th & 95th & 97th & 99th \\
\hline \multicolumn{17}{|l|}{ Boys } \\
\hline \multicolumn{17}{|c|}{ Elementary school } \\
\hline Grade 1 & 3,299 & $122.1 \pm 0.1$ & - & 110.1 & 112.6 & 114.0 & 115.8 & 116.9 & 118.5 & 121.8 & 125.3 & 127.3 & 128.4 & 130.5 & 132.1 & 135.4 \\
\hline Grade 2 & 3,357 & $127.8 \pm 0.1$ & 5.7 & 115.5 & 118.0 & 119.2 & 121.0 & 122.2 & 124.0 & 127.8 & 131.4 & 133.3 & 134.7 & 137.0 & 138.3 & 141.1 \\
\hline Grade 3 & 3,383 & $33.8 \pm 0.1$ & & 120.4 & 123.2 & 124.4 & 126.4 & 127.8 & 129.8 & 133.7 & 137.6 & 139.7 & 141.2 & 143.4 & 145.0 & 148.0 \\
\hline Grade 4 & 3,355 & $139.7 \pm 0.1$ & 5.9 & 126.2 & 128.8 & 130.0 & 131.9 & 133.3 & 135.4 & 139.4 & 143.6 & 145.9 & 147.9 & 150.3 & 152.1 & 154.8 \\
\hline Grade 5 & 3,384 & $145.0 \pm 0.1$ & 5.3 & 130.0 & 132.7 & 134.2 & 136.5 & 138.0 & 140.0 & 144.3 & 148.8 & 151.2 & 153.2 & 156.0 & 158.0 & 161.9 \\
\hline Grade 6 & 3,333 & $52.2 \pm 0.1$ & 7.2 & 135.9 & 138.8 & 140.5 & 142.8 & 144.5 & 147.0 & 151.9 & 157.0 & 160.3 & 162.1 & 165.0 & 167.0 & 170.5 \\
\hline \multicolumn{17}{|c|}{ Middle school } \\
\hline Grade 1 & 5,038 & $160.4 \pm 0.1$ & 8.2 & 141.5 & 145.0 & 147.2 & 150.0 & 151.8 & 154.9 & 160.5 & 166.0 & 168.5 & 170.1 & 172.7 & 174.3 & 177.4 \\
\hline Grade 2 & 5,102 & $166.3 \pm 0.1$ & 5.9 & 147.6 & 151.6 & 153.6 & 156.7 & 158.8 & 161.7 & 166.5 & 170.8 & 173.2 & 174.9 & 177.1 & 178.6 & 181.7 \\
\hline Grade 3 & 315 & $702+01$ & 3.9 & 54.2 & 157.4 & 159.5 & 162.3 & 164.0 & 166.1 & 170.3 & 174.2 & 176.3 & 178.0 & 180.1 & 181.5 & 184.4 \\
\hline \multicolumn{17}{|l|}{ High school } \\
\hline Grade 1 & 188 & $72.6 \pm 0.1$ & & 159.0 & 161.5 & 162.8 & 165.2 & 166.6 & 168.7 & 172.4 & 176.4 & 178.5 & 180.0 & 182.1 & 183.7 & 186.3 \\
\hline Grade 2 & 448 & $73.3 \pm 0.1$ & 0.7 & 159.3 & 162.4 & 164.0 & 165.9 & 167.2 & 169.3 & 173.1 & 177.0 & 179.3 & 180.8 & 183.0 & 184.6 & 187.4 \\
\hline Grade 3 & 837 & $73.8 \pm 0.1$ & 0.5 & 160.4 & 162.9 & 164.3 & 166.3 & 167.8 & 169.9 & 173.7 & 177.6 & 179.8 & 181.1 & 183.1 & 184.6 & 187.1 \\
\hline \multicolumn{17}{|l|}{ Girls } \\
\hline \multicolumn{17}{|c|}{ Elementary school } \\
\hline Grade 1 & 3,138 & $120.9 \pm 0.1$ & - & 109.7 & 111.7 & 112.7 & 114.5 & 115.6 & 117.4 & 120.8 & 124.2 & 126.4 & 127.7 & 129.8 & 131.2 & 134.1 \\
\hline Grade 2 & 3,180 & $126.8 \pm 0.1$ & & 114.4 & 117.0 & 118.0 & 120.0 & 121.3 & 123.0 & 126.6 & 130.1 & 132.2 & 133.4 & 135.5 & 137.2 & 140.5 \\
\hline Grade 3 & 3,149 & $132.4 \pm 0.1$ & 5.6 & 119.1 & 121.6 & 123.3 & 125.2 & 126.5 & 128.5 & 132.4 & 136.2 & 138.6 & 139.9 & 142.2 & 144.0 & 146.8 \\
\hline Grade 4 & 3,201 & $139.1 \pm 0.1$ & & 125.7 & 127.7 & 128.9 & 131.0 & 132.5 & 134.6 & 139.0 & 143.4 & 145.9 & 147.8 & 150.4 & 152.3 & 154.8 \\
\hline Grade 5 & 3,180 & $145.6 \pm 0.1$ & 6.5 & 130.1 & 133.0 & 134.4 & 136.7 & 138.1 & 140.6 & 145.7 & 150.4 & 153.1 & 154.7 & 157.1 & 158.4 & 161.0 \\
\hline Grade 6 & 3,132 & $152.2 \pm 0.1$ & 6.6 & 136.7 & 139.7 & 141.3 & 143.9 & 145.7 & 148.1 & 152.4 & 156.6 & 158.6 & 160.2 & 162.2 & 163.3 & 166.2 \\
\hline \multicolumn{17}{|c|}{ Middle school } \\
\hline Grade 1 & 4,785 & $156.9 \pm 0.1$ & 4.7 & 142.9 & 146.2 & 147.6 & 150.0 & 151.4 & 153.3 & 157.0 & 160.4 & 162.5 & 163.9 & 166.0 & 167.5 & 169.6 \\
\hline Grade 2 & 4,900 & $158.9 \pm 0.1$ & 2.0 & 146.5 & 149.0 & 150.2 & 152.2 & 153.5 & 155.4 & 158.9 & 162.4 & 164.4 & 165.7 & 167.7 & 169.0 & 171.3 \\
\hline Grade 3 & 5,010 & $160.3 \pm 0.1$ & 14 & 147.8 & 150.2 & 151.7 & 153.3 & 154.7 & 156.5 & 160.1 & 163.8 & 165.9 & 167.2 & 169.3 & 170.5 & 173.9 \\
\hline \multicolumn{17}{|l|}{ High school } \\
\hline Grade 1 & 5,839 & $160.9 \pm 0.1$ & 0.6 & 148.5 & 151.0 & 152.2 & 154.0 & 155.3 & 157.2 & 160.8 & 164.3 & 166.2 & 167.5 & 169.5 & 170.8 & 173.3 \\
\hline Grade 2 & 6,496 & $160.9 \pm 0.1$ & 0.0 & 148.3 & 151.0 & 152.5 & 154.2 & 155.6 & 157.4 & 160.8 & 164.4 & 166.2 & 167.5 & 169.6 & 171.0 & 173.4 \\
\hline Grade 3 & 6,905 & $160.9 \pm 0.1$ & 0.0 & 148.3 & 151.0 & 152.3 & 154.0 & 155.3 & 157.2 & 160.8 & 164.4 & 166.4 & 167.8 & 170.0 & 171.0 & 173.4 \\
\hline
\end{tabular}

$\mathrm{SE}$, standard error.

"Difference values are the mean differences in height between the corresponding grade-students and the 1-year younger gradestudents. 
and the 1 st year of middle school $(8.6 \mathrm{~cm} / \mathrm{yr}$ increase $)$ and for girls between the 5th and 6th grades of elementary school (6.7 $\mathrm{cm} / \mathrm{yr}$ increase).

\section{Percentile values of weight according to sex and school grade}

Table 3 presents the mean and percentile values of weight by sex and school grade. The 50th percentile values of the weight of 6th-grade elementary school boys and girls were $47.7 \mathrm{~kg}$ and $44.8 \mathrm{~kg}$, respectively. The 50th percentile values of the weight of $3 \mathrm{rd}$-year high school boys and girls were $68.9 \mathrm{~kg}$ and $55.8 \mathrm{~kg}$, respectively.

\section{Percentile values of BMl according to sex and school grade}

Table 4 presents the mean and percentile values of BMI according to sex and school grade. The 50th percentile values of BMI of 6th-grade elementary school boys and girls were 20.5 $\mathrm{kg} / \mathrm{m}^{2}$ and $19.2 \mathrm{~kg} / \mathrm{m}^{2}$, respectively. The 50th percentile values of BMI of 3rd-year high school boys and girls were $22.8 \mathrm{~kg} / \mathrm{m}^{2}$ and $21.6 \mathrm{~kg} / \mathrm{m}^{2}$, respectively. The 5th percentile values of BMI, which are associated with underweight status, were $18.0 \mathrm{~kg} / \mathrm{m}^{2}$ and $17.5 \mathrm{~kg} / \mathrm{m}^{2}$ for $3 \mathrm{rd}-$ year high school boys and girls, respectively. The 85th percentile values of BMI, which are associated with overweight, were $27.7 \mathrm{~kg} / \mathrm{m}^{2}$ and $25.8 \mathrm{~kg} / \mathrm{m}^{2}$ for 3 rd-year high school boys and girls, respectively. ${ }^{5)}$ The 95th percentile values of BMI, which are associated with obesity, were $31.9 \mathrm{~kg} / \mathrm{m}^{2}$ and $29.6 \mathrm{~kg} / \mathrm{m}^{2}$ for $3 \mathrm{rd}-$ year high school boys and girls, respectively.

Table 3. Mean and percentile values of weight by sex and school grade in Korean school students

\begin{tabular}{|c|c|c|c|c|c|c|c|c|c|c|c|c|c|c|c|c|}
\hline \multirow{2}{*}{ School grade } & \multirow{2}{*}{ No. } & \multirow{2}{*}{ Mean \pm SE } & \multirow{2}{*}{ Difference $^{*}$} & \multicolumn{13}{|c|}{ Percentile values of weight (kg) } \\
\hline & & & & $1 s t$ & $3 r d$ & 5 th & 10th & 15th & 25 th & 50th & 75th & 85th & 90th & 95th & 97th & 99th \\
\hline \multicolumn{17}{|l|}{ Boys } \\
\hline \multicolumn{17}{|c|}{ Elementary school } \\
\hline Grade 1 & 3,299 & $25.2 \pm 0.1$ & - & 17.4 & 18.8 & 19.2 & 20.0 & 20.7 & 21.7 & 24.0 & 27.6 & 29.8 & 32.0 & 35.6 & 37.8 & 42.4 \\
\hline Grade 2 & 3,357 & $28.9 \pm 0.2$ & 3.7 & 19.2 & 20.5 & 21.3 & 22.4 & 23.1 & 24.4 & 27.4 & 31.9 & 35.2 & 37.6 & 41.7 & 44.3 & 49.9 \\
\hline Grade 3 & 3,383 & $33.3 \pm 0.2$ & 4.4 & 21.4 & 22.8 & 23.7 & 25.0 & 26.0 & 27.5 & 31.5 & 37.5 & 41.5 & 44.1 & 48.8 & 52.3 & 58.2 \\
\hline Grade 4 & 3,355 & $37.9 \pm 0.2$ & 4.6 & 24.0 & 25.8 & 26.4 & 28.0 & 29.0 & 31.0 & 36.0 & 43.6 & 48.3 & 50.9 & 55.8 & 59.3 & 66.0 \\
\hline Grade 5 & 3,384 & $42.7 \pm 0.2$ & 4.8 & 26.1 & 28.2 & 29.1 & 30.9 & 32.2 & 34.5 & 40.6 & 48.5 & 53.3 & 56.9 & 63.1 & 66.7 & 74.1 \\
\hline Grade 6 & 3,333 & $49.1 \pm 0.3$ & 6.4 & 28.6 & 31.5 & 32.7 & 35.0 & 37.0 & 40.4 & 47.7 & 56.5 & 61.8 & 65.5 & 71.8 & 75.4 & 81.6 \\
\hline \multicolumn{17}{|c|}{ Middle school } \\
\hline Grade 1 & 5,038 & $54.6 \pm 0.2$ & 5.5 & 31.8 & 34.9 & 36.7 & 39.8 & 42.0 & 45.2 & 52.7 & 62.7 & 68.0 & 72.0 & 78.1 & 82.5 & 92.0 \\
\hline Grade 2 & 5,102 & $60.7 \pm 0.3$ & 6.1 & 36.0 & 39.9 & 41.7 & 44.9 & 47.3 & 50.8 & 58.3 & 68.5 & 75.4 & 79.6 & 87.2 & 92.0 & 102.0 \\
\hline Grade 3 & 5,315 & $64.6 \pm 0.2$ & 3.9 & 40.9 & 44.4 & 46.2 & 49.2 & 51.3 & 54.6 & 62.2 & 72.4 & 79.1 & 84.0 & 92.2 & 98.0 & 108.5 \\
\hline \multicolumn{17}{|l|}{ High school } \\
\hline Grade 1 & 6,188 & $67.8 \pm 0.2$ & 3.2 & 44.6 & 47.6 & 49.3 & 52.5 & 54.4 & 57.8 & 65.3 & 75.0 & 81.6 & 86.6 & 94.8 & 99.6 & 108.6 \\
\hline Grade 2 & 6,448 & $69.3 \pm 0.2$ & 1.5 & 46.1 & 49.1 & 51.0 & 53.8 & 55.9 & 59.5 & 66.9 & 76.7 & 83.2 & 87.8 & 96.6 & 102.3 & 112.9 \\
\hline Grade 3 & 6,837 & $71.3 \pm 0.2$ & 2.0 & 48.0 & 51.1 & 52.6 & 55.8 & 58.0 & 61.3 & 68.9 & 78.2 & 85.0 & 90.0 & 97.9 & 103.0 & 115.1 \\
\hline \multicolumn{17}{|c|}{ Girls } \\
\hline \multicolumn{17}{|c|}{ Elementary school } \\
\hline Grade 1 & 3,138 & $24.2 \pm 0.1$ & - & 17.0 & 18.0 & 18.6 & 19.3 & 20.0 & 21.0 & 23.4 & 26.5 & 28.5 & 30.3 & 32.6 & 34.9 & 39.6 \\
\hline Grade 2 & 3,180 & $27.4 \pm 0.1$ & 3.2 & 18.6 & 19.7 & 20.4 & 21.4 & 22.2 & 23.4 & 26.0 & 30.0 & 32.8 & 35.2 & 38.5 & 40.5 & 45.2 \\
\hline Grade 3 & 3,149 & $30.8 \pm 0.1$ & 3.4 & 20.4 & 21.8 & 22.5 & 23.7 & 24.6 & 26.2 & 29.8 & 34.5 & 37.3 & 39.7 & 43.6 & 46.1 & 50.5 \\
\hline Grade 4 & 3,201 & $35.3 \pm 0.2$ & 4.5 & 22.5 & 24.0 & 24.8 & 26.5 & 27.6 & 29.4 & 34.1 & 40.1 & 44.0 & 46.9 & 51.0 & 54.3 & 60.3 \\
\hline Grade 5 & 3,180 & $40.3 \pm 0.2$ & 5.0 & 24.7 & 26.9 & 28.0 & 29.9 & 31.2 & 33.7 & 39.0 & 45.7 & 49.5 & 52.6 & 57.2 & 60.6 & 67.2 \\
\hline Grade 6 & 3,132 & $46.0 \pm 0.3$ & 5.7 & 28.3 & 31.0 & 32.2 & 34.5 & 36.3 & 39.3 & 44.8 & 51.5 & 55.5 & 58.6 & 64.5 & 67.9 & 74.6 \\
\hline \multicolumn{17}{|c|}{ Middle school } \\
\hline Grade 1 & 4,785 & $50.4 \pm 0.2$ & 4.4 & 32.3 & 35.4 & 36.9 & 39.2 & 40.8 & 43.5 & 49.0 & 55.8 & 60.3 & 64.1 & 70.6 & 74.4 & 80.6 \\
\hline Grade 2 & 4,900 & $53.2 \pm 0.2$ & 2.8 & 36.0 & 38.5 & 40.0 & 42.2 & 44.0 & 46.3 & 51.9 & 58.2 & 63.0 & 67.0 & 73.1 & 77.1 & 87.0 \\
\hline Grade 3 & 5,010 & $55.4 \pm 0.2$ & 2.2 & 38.9 & 41.1 & 42.5 & 44.3 & 45.9 & 48.1 & 53.4 & 60.0 & 64.8 & 68.4 & 75.0 & 79.6 & 88.8 \\
\hline \multicolumn{17}{|l|}{ High school } \\
\hline Grade 1 & 5,839 & $56.7 \pm 0.2$ & 1.3 & 39.7 & 42.0 & 43.3 & 45.5 & 47.1 & 49.6 & 54.8 & 61.5 & 66.0 & 70.0 & 76.3 & 80.9 & 90.2 \\
\hline Grade 2 & 6,496 & $57.0 \pm 0.2$ & 0.3 & 40.0 & 42.4 & 43.9 & 46.0 & 47.6 & 50.0 & 55.0 & 61.9 & 66.8 & 70.7 & 77.5 & 82.0 & 90.6 \\
\hline Grade 3 & 6,905 & $57.5 \pm 0.2$ & 0.5 & 40.0 & 42.8 & 44.2 & 46.5 & 48.0 & 50.4 & 55.8 & 62.9 & 67.7 & 71.5 & 78.0 & 82.2 & 92.7 \\
\hline
\end{tabular}

SE, standard error.

"Difference values are the mean differences in weight between the corresponding grade-students and the 1-year younger gradestudents. 
Table 4. Mean and percentile values of body mass index by sex and school grade in Korean school students

\begin{tabular}{|c|c|c|c|c|c|c|c|c|c|c|c|c|c|c|c|c|}
\hline \multirow{2}{*}{ School grade } & \multirow{2}{*}{ No. } & \multirow{2}{*}{ Mean \pm SE } & \multirow{2}{*}{ Difference $^{*}$} & \multicolumn{13}{|c|}{ Percentile values of body mass index $\left(\mathrm{kg} / \mathrm{m}^{2}\right)$} \\
\hline & & & & $1 \mathrm{st}$ & $3 r d$ & 5 th & 10th & 15th & 25 th & 50th & 75th & 85th & 90th & 95th & 97th & 99th \\
\hline \multicolumn{17}{|l|}{ Boys } \\
\hline \multicolumn{17}{|c|}{ Elementary school } \\
\hline Grade 1 & 3,299 & $16.8 \pm 0.1$ & - & 13.0 & 13.6 & 13.9 & 14.4 & 14.6 & 15.1 & 16.2 & 18.0 & 19.2 & 20.3 & 22.1 & 23.4 & 25.2 \\
\hline Grade 2 & 3,357 & $17.5 \pm 0.1$ & 0.7 & 13.2 & 13.8 & 14.1 & 14.6 & 15.0 & 15.5 & 16.7 & 18.9 & 20.6 & 21.8 & 23.4 & 24.8 & 26.6 \\
\hline Grade 3 & 3,383 & $18.5 \pm 0.1$ & 1.0 & 13.3 & 14.0 & 14.4 & 15.0 & 15.3 & 15.9 & 17.7 & 20.4 & 22.0 & 23.3 & 25.3 & 26.5 & 28.9 \\
\hline Grade 4 & 3,355 & $19.3 \pm 0.1$ & 0.8 & 13.8 & 14.4 & 14.8 & 15.3 & 15.8 & 16.5 & 18.5 & 21.6 & 23.2 & 24.5 & 26.4 & 27.5 & 30.1 \\
\hline Grade 5 & 3,384 & $20.1 \pm 0.1$ & 0.8 & 14.0 & 14.8 & 15.2 & 15.8 & 16.3 & 17.1 & 19.4 & 22.5 & 24.4 & 25.5 & 27.4 & 28.8 & 30.9 \\
\hline Grade 6 & 3,333 & $21.0 \pm 0.1$ & 0.9 & 14.5 & 15.1 & 15.6 & 16.3 & 16.9 & 17.9 & 20.5 & 23.7 & 25.5 & 26.6 & 28.4 & 29.6 & 32.0 \\
\hline \multicolumn{17}{|c|}{ Middle school } \\
\hline Grade 1 & 5,038 & $21.1 \pm 0.1$ & 0.1 & 14.6 & 15.4 & 15.8 & 16.5 & 17.1 & 18.0 & 20.4 & 23.7 & 25.6 & 26.8 & 28.7 & 30.0 & 32.8 \\
\hline Grade 2 & 5,102 & $21.8 \pm 0.1$ & 0.7 & 15.1 & 15.9 & 16.4 & 17.2 & 17.8 & 18.7 & 21.0 & 24.4 & 26.5 & 28.0 & 30.1 & 31.6 & 34.1 \\
\hline Grade 3 & 5,315 & $22.2 \pm 0.1$ & 0.4 & 15.7 & 16.4 & 16.9 & 17.5 & 18.1 & 19.1 & 21.4 & 24.7 & 26.8 & 28.4 & 30.7 & 32.4 & 35.2 \\
\hline \multicolumn{17}{|l|}{ High school } \\
\hline Grade 1 & 6,188 & $22.7 \pm 0.1$ & 0.5 & 16.0 & 16.8 & 17.3 & 18.0 & 18.6 & 19.6 & 21.9 & 25.1 & 27.1 & 28.6 & 30.9 & 32.5 & 35.6 \\
\hline Grade 2 & 6,448 & $23.0 \pm 0.1$ & 0.3 & 16.1 & 17.0 & 17.5 & 18.3 & 19.0 & 20.0 & 22.3 & 25.2 & 27.3 & 29.0 & 31.3 & 33.2 & 36.4 \\
\hline Grade 3 & 6,837 & $23.6 \pm 0.1$ & 0.6 & 16.7 & 17.5 & 18.0 & 18.8 & 19.4 & 20.5 & 22.8 & 25.8 & 27.7 & 29.3 & 31.9 & 33.7 & 37.0 \\
\hline
\end{tabular}

\section{Girls}

\begin{tabular}{cccccccccccccccccc}
\hline \multicolumn{2}{c}{$\begin{array}{c}\text { Elementary school } \\
\text { Grade 1 }\end{array}$} & 3,138 & $16.4 \pm 0.0$ & - & 12.8 & 13.4 & 13.6 & 14.1 & 14.4 & 14.9 & 16.0 & 17.6 & 18.6 & 19.5 & 20.9 & 22.0 & 23.7 \\
Grade 2 & 3,180 & $16.9 \pm 0.1$ & 0.5 & 12.9 & 13.4 & 13.7 & 14.2 & 14.5 & 15.1 & 16.3 & 18.1 & 19.5 & 20.5 & 21.9 & 22.9 & 25.1 \\
Grade 3 & 3,149 & $17.5 \pm 0.1$ & 0.6 & 13.2 & 13.7 & 14.0 & 14.5 & 14.8 & 15.4 & 17.0 & 19.1 & 20.3 & 21.3 & 23.0 & 23.8 & 25.8 \\
Grade 4 & 3,201 & $18.1 \pm 0.1$ & 0.6 & 13.1 & 13.7 & 14.1 & 14.7 & 15.1 & 15.8 & 17.6 & 20.0 & 21.5 & 22.7 & 24.1 & 25.2 & 27.6 \\
Grade 5 & 3,180 & $18.9 \pm 0.1$ & 0.8 & 13.5 & 14.2 & 14.6 & 15.2 & 15.7 & 16.4 & 18.3 & 20.8 & 22.4 & 23.6 & 25.2 & 26.3 & 28.6 \\
Grade 6 & 3,132 & $19.7 \pm 0.1$ & 0.8 & 14.0 & 14.7 & 15.2 & 15.8 & 16.4 & 17.2 & 19.2 & 21.7 & 23.3 & 24.3 & 26.2 & 27.6 & 29.9 \\
Middle school & & & & & & & & & & & & & & & \\
Grade 1 & 4,785 & $20.4 \pm 0.1$ & 0.7 & 14.5 & 15.3 & 15.7 & 16.5 & 17.1 & 18.0 & 19.9 & 22.3 & 24.0 & 25.4 & 27.5 & 28.9 & 31.5 \\
Grade 2 & 4,900 & $21.0 \pm 0.1$ & 0.6 & 15.2 & 15.9 & 16.4 & 17.2 & 17.8 & 18.6 & 20.4 & 22.8 & 24.6 & 25.9 & 28.3 & 29.6 & 32.7 \\
Grade 3 & 5,010 & $21.5 \pm 0.1$ & 0.5 & 15.7 & 16.5 & 16.9 & 17.6 & 18.2 & 19.0 & 20.8 & 23.3 & 24.9 & 26.3 & 28.4 & 30.0 & 33.4 \\
High school & & & & & & & & & & & & & & & \\
Grade 1 & 5,839 & $21.9 \pm 0.1$ & 0.4 & 16.0 & 16.8 & 17.2 & 18.0 & 18.5 & 19.3 & 21.2 & 23.5 & 25.2 & 26.6 & 29.0 & 30.6 & 33.4 \\
Grade 2 & 6,496 & $22.0 \pm 0.1$ & 0.1 & 16.1 & 17.0 & 17.5 & 18.1 & 18.6 & 19.5 & 21.3 & 23.7 & 25.4 & 27.0 & 29.4 & 30.8 & 34.2 \\
Grade 3 & 6,905 & $22.2 \pm 0.1$ & 0.2 & 16.2 & 17.1 & 17.5 & 18.3 & 18.8 & 19.6 & 21.6 & 24.1 & 25.8 & 27.2 & 29.6 & 31.4 & 34.7 \\
\hline
\end{tabular}

SE, standard error.

"Difference values are the mean differences in body mass index between the corresponding grade-students and the 1-year younger grade-students.

\section{Comparison of height and weight values according to sex and school grade between KSHES 2010 and 2018}

Table 5 and Fig. 1 present the comparison of mean height and weight values between KSHES 2010 and 2018. The mean height values of students between the 3rd grade in elementary school and the 1st year in high school were significantly higher in 2018 compared with those in 2010 , in both sexes. In boys, the grade that showed the greatest height gap was the 1st year of middle school, where the difference in mean height values was $2.5 \mathrm{~cm}$ $(P<0.0001)$. In girls, the grade that showed the greatest height gap was also the 1st year of middle school, where the difference was $1.4 \mathrm{~cm}(P<0.0001)$. Additionally, the mean weight values of the study population for most school grades in 2018 were significantly higher than those in 2010 among both boys and girls $(P<0.05)$.

\section{Discussion}

This study presented the mean and selected percentile values of height, weight, and BMI of Korean school students based on data from the most recent large-scale measurement. In 2018, the mean height among students between the 3rd grade in elementary school and the 1st year in high school was significantly taller than those in 2010 for both sexes. The mean heights of the 3rd-year high school students in 2018 were 173.8 $\mathrm{cm}$ and $160.9 \mathrm{~cm}$ for boys and girls, respectively. No significant differences in the mean heights of 1 st and 2 nd graders in elementary school and seniors in high school were found between 2010 and 2018 for either sex. Meanwhile, the mean weight in 2018 was significantly higher than in 2010 for most school grades.

The mean heights of the 3rd-year high school students in 
Table 5. Comparison of mean height and weight by sex and school grade between Korea School Health Examination Survey (KSHES) 2010 $(n=188,352)$ and $2018(n=107,954)$

\begin{tabular}{|c|c|c|c|c|c|c|c|c|c|c|c|c|}
\hline \multirow{2}{*}{ School grade } & \multicolumn{2}{|c|}{ KSHES 2010} & \multicolumn{2}{|c|}{ KSHES 2018} & \multirow{2}{*}{ Difference } & \multirow{2}{*}{$P$-value } & \multicolumn{2}{|c|}{ KSHES 2010} & \multicolumn{2}{|c|}{ KSHES 2018} & \multirow{2}{*}{ Difference } & \multirow{2}{*}{$P$-value } \\
\hline & No. & Height $(\mathrm{cm})$ & No. & Height $(\mathrm{cm})$ & & & No. & Weight (kg) & No. & Weight $(\mathrm{kg})$ & & \\
\hline \multicolumn{13}{|l|}{ Boys } \\
\hline \multicolumn{13}{|c|}{ Elementary school } \\
\hline Grade 1 & 7,487 & $121.8 \pm 0.1$ & 3,299 & $122.1 \pm 0.1$ & 0.3 & 0.0694 & 7,488 & $24.9 \pm 0.1$ & 3,299 & $25.2 \pm 0.1$ & 0.3 & 0.0694 \\
\hline Grade 2 & 7,476 & $127.7 \pm 0.1$ & 3,357 & $127.8 \pm 0.1$ & 0.1 & 0.5393 & 7,475 & $28.4 \pm 0.1$ & 3,357 & $28.9 \pm 0.2$ & 0.5 & 0.0125 \\
\hline Grade 3 & 7,578 & $133.1 \pm 0.1$ & 3,383 & $133.8 \pm 0.1$ & 0.7 & $<0.0001$ & 7,576 & $32.1 \pm 0.1$ & 3,383 & $33.3 \pm 0.2$ & 1.2 & $<0.0001$ \\
\hline Grade 4 & 7,618 & $138.4 \pm 0.1$ & 3,355 & $139.7 \pm 0.1$ & 1.3 & $<0.0001$ & 7,617 & $35.9 \pm 0.1$ & 3,355 & $37.9 \pm 0.2$ & 2.0 & $<0.0001$ \\
\hline Grade 5 & 7,598 & $144.0 \pm 0.1$ & 3,384 & $145.0 \pm 0.1$ & 1.0 & $<0.0001$ & 7,594 & $41.1 \pm 0.1$ & 3,384 & $42.7 \pm 0.2$ & 1.6 & $<0.0001$ \\
\hline Grade 6 & 7,669 & $150.2 \pm 0.1$ & 3,333 & $152.2 \pm 0.1$ & 2.0 & $<0.0001$ & 7,669 & $46.1 \pm 0.1$ & 3,333 & $49.1 \pm 0.3$ & 3.0 & $<0.0001$ \\
\hline \multicolumn{13}{|c|}{ Middle school } \\
\hline Grade 1 & 9,389 & $157.9 \pm 0.1$ & 5,038 & $160.4 \pm 0.1$ & 2.5 & $<0.0001$ & 9,388 & $51.7 \pm 0.2$ & 5,038 & $54.6 \pm 0.2$ & 2.9 & $<0.0001$ \\
\hline Grade 2 & 9,294 & $164.2 \pm 0.1$ & 5,102 & $166.3 \pm 0.1$ & 2.1 & $<0.0001$ & 9,290 & $57.2 \pm 0.2$ & 5,102 & $60.7 \pm 0.3$ & 3.5 & $<0.0001$ \\
\hline Grade 3 & 9,107 & $168.9 \pm 0.1$ & 5,315 & $170.2 \pm 0.1$ & 1.3 & $<0.0001$ & 9,106 & $61.5 \pm 0.2$ & 5,315 & $64.6 \pm 0.2$ & 3.1 & $<0.0001$ \\
\hline \multicolumn{13}{|l|}{ High school } \\
\hline Grade 1 & 9,220 & $171.8 \pm 0.1$ & 6,188 & $172.6 \pm 0.1$ & 0.8 & $<0.0001$ & 9,219 & $64.7 \pm 0.2$ & 6,188 & $67.8 \pm 0.2$ & 3.1 & $<0.0001$ \\
\hline Grade 2 & 8,952 & $173.0 \pm 0.1$ & 6,448 & $173.3 \pm 0.1$ & 0.3 & 0.0383 & 8,952 & $66.6 \pm 0.2$ & 6,448 & $69.3 \pm 0.2$ & 2.7 & $<0.0001$ \\
\hline Grade 3 & 8,884 & $173.7 \pm 0.1$ & 6,837 & $173.8 \pm 0.1$ & 0.1 & 0.4868 & 8,884 & $68.1 \pm 0.2$ & 6,837 & $71.3 \pm 0.2$ & 3.2 & $<0.0001$ \\
\hline \multicolumn{13}{|l|}{ Girls } \\
\hline \multicolumn{13}{|c|}{ Elementary school } \\
\hline Grade 1 & 6,955 & $120.6 \pm 0.1$ & 3,138 & $120.9 \pm 0.1$ & 0.3 & 0.0652 & 6,955 & $23.7 \pm 0.1$ & 3,138 & $24.2 \pm 0.1$ & 0.5 & 0.0021 \\
\hline Grade 2 & 6,810 & $126.4 \pm 0.1$ & 3,180 & $126.8 \pm 0.1$ & 0.4 & 0.0136 & 6,809 & $26.9 \pm 0.1$ & 3,180 & $27.4 \pm 0.1$ & 0.5 & 0.0020 \\
\hline Grade 3 & 7,025 & $132.0 \pm 0.1$ & 3,149 & $132.4 \pm 0.1$ & 0.4 & 0.0147 & 7,024 & $30.3 \pm 0.1$ & 3,149 & $30.8 \pm 0.1$ & 0.5 & 0.0023 \\
\hline Grade 4 & 7,162 & $138.1 \pm 0.1$ & 3,201 & $139.1 \pm 0.1$ & 1.0 & $<0.0001$ & 7,161 & $34.2 \pm 0.1$ & 3,201 & $35.3 \pm 0.2$ & 1.1 & $<0.0001$ \\
\hline Grade 5 & 7,024 & $144.6 \pm 0.1$ & 3,180 & $145.6 \pm 0.1$ & 1.0 & $<0.0001$ & 7,023 & $39.2 \pm 0.1$ & 3,180 & $40.3 \pm 0.2$ & 1.1 & $<0.0001$ \\
\hline Grade 6 & 7,026 & $151.2 \pm 0.1$ & 3,132 & $152.2 \pm 0.1$ & 1.0 & $<0.0001$ & 7,024 & $44.4 \pm 0.1$ & 3,132 & $46.0 \pm 0.3$ & 1.6 & $<0.0001$ \\
\hline \multicolumn{13}{|c|}{ Middle school } \\
\hline Grade 1 & 7,839 & $155.5 \pm 0.1$ & 4,785 & $156.9 \pm 0.1$ & 1.4 & $<0.0001$ & 7,838 & $48.2 \pm 0.2$ & 4,785 & $50.4 \pm 0.2$ & 2.2 & $<0.0001$ \\
\hline Grade 2 & 7,501 & $158.2 \pm 0.1$ & 4,900 & $158.9 \pm 0.1$ & 0.7 & $<0.0001$ & 7,500 & $51.5 \pm 0.2$ & 4,900 & $53.2 \pm 0.2$ & 1.7 & 0.0332 \\
\hline Grade 3 & 7,496 & $159.6 \pm 0.1$ & 5,010 & $160.3 \pm 0.1$ & 0.7 & $<0.0001$ & 7,496 & $53.2 \pm 0.1$ & 5,010 & $55.4 \pm 0.2$ & 2.2 & $<0.0001$ \\
\hline \multicolumn{13}{|l|}{ High school } \\
\hline Grade 1 & 7,395 & $160.6 \pm 0.1$ & 5,839 & $160.9 \pm 0.1$ & 0.3 & 0.0361 & 7,395 & $54.9 \pm 0.2$ & 5,839 & $56.7 \pm 0.2$ & 1.8 & $<0.0001$ \\
\hline Grade 2 & 7,094 & $160.7 \pm 0.1$ & 6,496 & $160.9 \pm 0.1$ & 0.2 & 0.1585 & 7,093 & $55.5 \pm 0.2$ & 6,496 & $57.0 \pm 0.2$ & 1.5 & $<0.0001$ \\
\hline Grade 3 & 7,217 & $160.9 \pm 0.1$ & 6,905 & $160.9 \pm 0.1$ & 0.0 & 1.0000 & 7,217 & $55.6 \pm 0.2$ & 6,905 & $57.5 \pm 0.2$ & 1.9 & $<0.0001$ \\
\hline
\end{tabular}

Values are presented as mean \pm standard error.

2018, $173.8 \mathrm{~cm}$ for boys and $160.9 \mathrm{~cm}$ for girls, were found to be similar to those in 2010. However, the mean height of the age group from the 3 rd grade in elementary school through the 3rd year in middle school was significantly taller in 2018 than in 2010, showing differences of about $2 \mathrm{~cm}$ for boys and $1 \mathrm{~cm}$ for girls. This result suggests that the heights during the rapid growth period are taller than in the past, while near-final height values are similar to those of the past.

A previous study revealed a significant increment in the mean height among the Korean pediatric population between 1965 and 2005. ${ }^{7)}$ However, another study demonstrated stabilized trends in the near-final height of Korean adolescents after comparing the Korean national anthropometric data in 2005 and $2010 .{ }^{8)}$ The increase in the height of pediatric adolescents has reportedly slowed down in most developed countries from the end of the 20th century since the rapid economic growth has ceased and the overall living environment has stabilized. ${ }^{7,910)}$
Our study results are consistent with the previous study, indicating no significant changes in near-final height over the past decade, similar to other developed countries. ${ }^{7,910)}$

While there is little difference in the mean height values between 2010 and 2018 in the younger age groups, a significant difference was observed beginning from the 6th grade in elementary school for boys and the 4th grade in elementary school for girls. The growth spurt is followed by the appearance of secondary sexual characteristics during puberty. ${ }^{11)}$ Therefore, consistent with several previous epidemiological studies, our findings reflect the accelerated timing of puberty and the growth spurt among Korean school students in recent decades. ${ }^{3,411-14)}$

The mean weight in 2018 was found to be significantly higher compared to 2010 in both sexes through all of the school grades, except for 1st-grade elementary school boys. This finding suggests that the rapid increase in the mean weight of the Korean pediatric population, which has been observed since the 

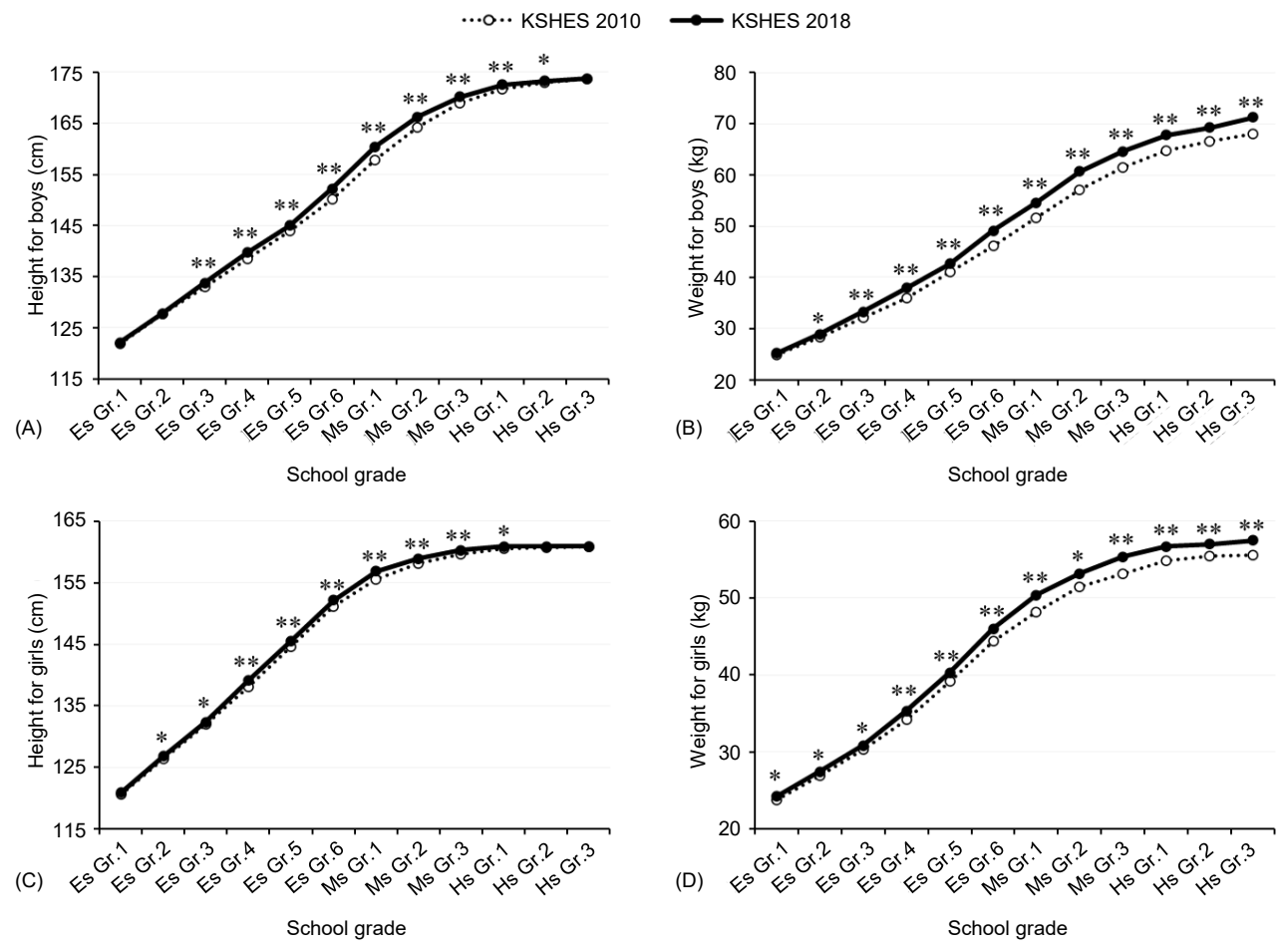

Fig. 1. Comparisons of mean height and weight of Korean school students by sex and school grade between Korea School Health Examination Surveys (KSHES) from 2010 and 2018. (A) Mean height for boys, (B) mean weight for boys, (C) mean height for girls, and (D) mean weight for girls. Es, elementary school; Gr, grade; Ms, middle school; Hs, high school. ${ }^{*} P<0.05 .{ }^{* *} P<0.001$.

1980s, continues into 2018. ${ }^{7)}$

In the present study, the 50th percentile values of BMI of the $3 \mathrm{rd}$-year high school students were $22.8 \mathrm{~kg} / \mathrm{m}^{2}$ and $21.6 \mathrm{~kg} /$ $\mathrm{m}^{2}$ for boys and girls, respectively. On the other hand, the 50th percentile values of BMI for the same age group in the KNGC 2017 , about $22.3 \mathrm{~kg} / \mathrm{m}^{2}$ in boys and about $21.0 \mathrm{~kg} / \mathrm{m}^{2}$ in girls, were approximately $0.5 \mathrm{~kg} / \mathrm{m}^{2}$ lower than those in the current study population. ${ }^{5}$ In this study, the 95th percentile values of BMI were $31.9 \mathrm{~kg} / \mathrm{m}^{2}$ and $29.6 \mathrm{~kg} / \mathrm{m}^{2}$ for 3 rd-year high school boys and girls, respectively. These values exceeded the BMI 99th percentile values for adolescents in the same age group in the KNGC 2017 (28.9 kg/m $\mathrm{m}^{2}$ in boys and $27.7 \mathrm{~kg} / \mathrm{m}^{2}$ in girls). ${ }^{5)}$ This finding indicates an increasing prevalence of extreme obesity among Korean school students, especially among older students.

This study has certain limitations. First, although the Ministry of Education and the Korea Educational Environments Protection Agency provided a standard protocol for anthropometric measurements, measurement bias may exist since the measuring devices were not standardized. Second, the survey did not include questionnaires on pubertal signs. Third, due to the absence of BMI reference values in the KSHES 2010 result report, we could not compare the BMI values between the 2 datasets in this study. Nevertheless, the strength of this study is that it was based on the most recent, large-scale, and valid measurements of anthropometric indices.

In conclusion, this study demonstrated the current status of anthropometric index measurements among Korean school students based on the latest large-scale data in 2018 and also compared them with previous data from 2010.

No difference in height was observed between 2010 and 2018 for 3rd-year high school students, although height values were significantly higher in 2018 than in 2010 among school students undergoing a rapid growth period. Meanwhile, weight values in 2018 were significantly higher than in 2010 in nearly all of the school grades. Therefore, the KSHES might be useful in providing consistently crucial information of anthropometric indices to modify and establish the KNGC in the future.

\section{Ethical statement}

This study was approved by the Institutional Review Board (IRB) of the Inje University Sanggye Paik Hospital (approval number: SGPAIK 2020-02-002). The data are governmentdesignated statistics (approval number: 112002) based on Article 17 of the Statistics Act.

\section{Conflict of interest}

No potential conflict of interest relevant to this article was reported. 


\section{Acknowledgments}

We are grateful to the Ministry of Education of South Korea for permission to use the KSHES data. This study is based on the KSHES, which was conducted by the Ministry of Education with funding from the Government of Korea.

\section{References}

1. Granados A, Gebremariam A, Lee JM. Relationship between timing of peak height velocity and pubertal staging in boys and girls. J Clin Res Pediatr Endocrinol 2015;7:2357.

2. Moon JS, Lee SY, Nam CM, Choi JM, Choe BK, Seo JW, et al. 2007 Korean National Growth Charts: review of developmental process and an outlook. Korean J Pediatr 2008;51:1-25.

3. Kim SH, Huh K, Won S, Lee KW, Park MJ. A Significant Increase in the Incidence of Central Precocious Puberty among Korean Girls from 2004 to 2010. PLoS One 2015;10:e0141844.

4. Kim YJ, Kwon A, Jung MK, Kim KE, Suh J, Chae HW, et al. Incidence and prevalence of central precocious puberty in Korea: an epidemiologic study based on a National Database. J Pediatr 2019;208:221-8.

5. Kim JH, Yun S, Hwang SS, Shim JO, Chae HW, Lee YJ, et al. The 2017 Korean National Growth Charts for children and adolescents: development, improvement, and prospects. Korean J Pediatr 2018;61:135-49.

6. Ministry of Education and Korea Educational Environments Protection Agency. The Korea School Health
Examination Survey (KSHES) 2018 [Internet]. Cheongju (Korea): Ministry of Education and Korea Educational Environments Protection Agency; [cited 2019 Nov 13]. Available from: http://www.schoolhealth.kr.

7. Kim JY, Oh IH, Lee EY, Choi KS, Choe BK, Yoon TY, et al. Anthropometric changes in children and adolescents from 1965 to 2005 in Korea. Am J Phys Anthropol 2008;136:2306.

8. Moon JS. Secular trends of body sizes in Korean children and adolescents: from 1965 to 2010 . Korean J Pediatr 2011;54:436-42.

9. Zellner K, Jaeger U, Kromeyer-Hauschild K. Height, weight and BMI of schoolchildren in Jena, Germany--are the secular changes levelling off? Econ Hum Biol 2004;2:28194.

10. Hauspie RC, Vercauteren M, Susanne C. Secular changes in growth and maturation: an update. Acta Paediatr Suppl 1997;423:20-7.

11. Chae HW, Suh I, Kwon AR, Kim YJ, Kim YH, Kang DR, et al. Longitudinal standards for height and height velocity in Korean children and adolescents: the Kangwha study. [corrected]. J Korean Med Sci 2013;28:1512-7.

12. Sørensen K, Mouritsen A, Aksglaede L, Hagen CP, Mogensen SS, Juul A. Recent secular trends in pubertal timing: implications for evaluation and diagnosis of precocious puberty. Horm Res Paediatr 2012;77:137-45.

13. Kim SH, Park MJ. Childhood obesity and pubertal development. Pediatr Gastroenterol Hepatol Nutr 2012;15:151-9.

14. Park MJ, Lee IS, Shin EK, Joung H, Cho SI. The timing of sexual maturation and secular trends of menarchial age in Korean adolescents. Korean J Pediatr 2006;49:610-6. 